\title{
Metal artefacts in MRI-guided brachytherapy of cervical cancer
}

\author{
Abraam S. Soliman, PhD'.2, Amir Owrangi, PhD'.3, Ananth Ravi, PhD',2,3, William Y. Song, PhD',2,3 \\ 'Department of Medical Physics, Sunnybrook Health Sciences Centre, Toronto, ${ }^{2}$ Physical Sciences Platform, Sunnybrook Research Institute, \\ Toronto, ${ }^{3}$ Department of Radiation Oncology, University of Toronto, Toronto, Ontario, Canada
}

\begin{abstract}
The importance of assessing the metal-induced artefacts in magnetic resonance imaging (MRI)-guided brachytherapy is growing along with the increasing interest of integrating MRI into the treatment procedure of cervical cancer. Examples of metal objects in use include intracavitary cervical applicators and interstitial needles. The induced artefacts increase the uncertainties in the clinical workflow and can be a potential obstacle for the accurate delivery of the treatment. Overcoming this problem necessitates a good understanding of its originating sources. Several efforts are recorded in the literature to quantify the extent of such artefacts, in phantoms and in clinical practice. Here, we elaborate on the origin of metal-induced artefacts in the light of brachytherapy applications, while summarizing recent efforts that have been made to assess and overcome the induced distortions.

Key words: brachytherapy, cervix cancer, cervical cancer, MRI.

\section{Purpose}

In the last two decades, magnetic resonance imaging (MRI) has become an indispensable tool used to diagnose, treat and monitor gynecological cancers $[1,2,3,4,5,6,7,8,9$, $10,11,12,13,14,15]$. The increasing access to MRI in radiation oncology departments, and its superior soft tissue contrast have favored it over other imaging modalities that have dominated cancer imaging for many years, such as ultrasound (US) and computed tomography (CT). The significant improvement in target/organ delineation offered by volumetric MR images can lead to improved treatment planning and delivery, better local control, and recently reported, higher survival rates $[7,8,9,10,11,14,16,17]$. However, MRI also comes with its own technical and logistical challenges such as increased cost, sophisticated patient care workflow, geometric imperfections, patient-related distortions, and its restrictive compatibility with magnetic materials $[1,4,14,18,19,20,21,22]$. The latter concern is particularly important for MRI-guided brachytherapy in which metallic objects, such as cervical applicators and titanium needles are used. Metal objects induce artefacts in the acquired MR images, which can degrade the quality of the image and/or its clinical usability $[1,5,19,23,24,25,26,27]$. Consequently, this might affect the potential improvement in the treatment planning expected from integrating MRI into the workflow. It is therefore important to understand the fundamentals of metal artefacts and their potential effect on brachytherapy treatment in order to maximize the benefits from MRI and enhance the treatment outcome.

The purpose of this review is to summarize the consequences of using metal objects in brachytherapy treatment and explain the rationale behind the expected artefacts, as well as the recent efforts to evaluate and/or overcome them. We will focus on cervical cancer, which constitutes a major application for MRI-guided brachytherapy.

\section{Technical aspects: why do we expect artefacts?}

Metal objects do not produce detectable signal in conventional MR images (i.e., they show up as signal voids). Nevertheless, when they are introduced into a static field, they can potentially interact differently than human tissue. The type of interaction can range from minor artefact in the acquired MR image to serious safety hazards as a result of strong translational forces and torques induced in the object [28]. In order to quantitatively describe the response of a material to the magnetic field, the concept of magnetic susceptibility must be introduced:

The volume susceptibility of a material is dimensionless and usually referred to by $X$, where $\chi_{0}$ is often used for the susceptibility of water $\left(\chi_{0}=-9.05 \mathrm{ppm}\right)$ [25]. Materials with negative $X$ are diamagnetic while paramagnetic materials have positive susceptibility values. The induced forces and torques of materials with $|\chi|<\sim 10,000 \mathrm{ppm}$ are usually manageable; nevertheless, they must be tested before MRI use [25]. It is important here to notice that the
Address for correspondence: Abraam Soliman, PhD, Physical Sciences Platform, Sunnybrook Research Institute, Sunnybrook Health Sciences Centre, 2075 Bayview Ave, M-wing, M6-612, Toronto, ON, Canada, phone: +1 416-480-6100, e-mail: abraam.soliman@sunnybrook.ca
Received: 07.06.2016

Accepted: 28.07 .2016

Published: 24.08.2016 
level of compatibility/safety of a material with MRI is determined based on multiple aspects and tests other than straightforward susceptibility values [28,29]. The susceptibility values of common materials used in brachytherapy are summarized in Table 1.

When a material is exposed to an external magnetic field it becomes magnetized; its magnetization is proportional to the magnetic susceptibility of the material and the strength of the applied magnetic field at the point where the material is placed [25]. Mathematically this can be expressed by:

$$
M=\chi H,
$$

where $M$ is the induced magnetization, $\chi$ is its susceptibility and $H$ is the applied magnetic field. Intuitively, when a foreign object is inserted in the region of MR magnetic field, it changes the resultant magnetization. For instance, if a needle is inserted during cervical brachytherapy treatment, it will induce a change in the magnetization proportional to the change in the susceptibility such that $\Delta \chi=\chi_{\text {needle }}-\chi_{\text {tissue }}$. Therefore, from MR perspective, needles with the same susceptibility as the surrounding tissue would be ideal for such applications rather than needles with zero susceptibility. In practice, however, all the metal objects used in brachytherapy applications are paramagnetic and have larger susceptibilities than that of human tissues. Once magnetized, the object produces a magnetic field that opposes the applied field and hence distort the initial magnetization. This distortion also varies with the shape and orientation of the object with respect to the main field, B0. As MR image acquisition relies mainly on predetermined magnetic field variations, these metal-induced distortions in the magnetic field will consequently induce artefacts in the received image, as described next.

\section{Technical aspects: image distortions}

In order to understand the significance of metal artefacts in brachytherapy applications, it is important to grasp the MR principles lying behind such distortions.

Table 1. Susceptibilities of water, tissue, and selected materials $[25,44,49,68]$

\begin{tabular}{lcc} 
Material & $\begin{array}{c}\text { Density } \\
\left(\mathrm{g} / \mathrm{cm}^{3}\right)\end{array}$ & $\begin{array}{c}\text { Susceptibility } \\
(\mathrm{ppm})\end{array}$ \\
\hline Gold & 19.3 & -34 \\
\hline PEEK & 1.3 & -9.33 \\
\hline Water $\left(37^{\circ}\right)$ & 0.933 & -9.05 \\
\hline Human tissues & $\sim 0.92-1.05$ & $\sim(-11.0$ to -7.0$)$ \\
\hline Air (NTP) & $1.29 \times 10^{-3}$ & 0.36 \\
\hline Aluminum & 2.7 & $20.7-20.9$ \\
\hline Tungsten & 19.3 & $77.2-80$ \\
\hline Titanium & 4.54 & 182 \\
\hline $\begin{array}{l}\text { Stainless steel } \\
\text { (nonmagnetic, austenitic) }\end{array}$ & 8.0 & $3520-6700$
\end{tabular}

PEEK - polyether ether ketone, NTP - normal temperature $\left[20^{\circ} \mathrm{C}\right]$ and pressure [101.325 kPa]
The objects placed within the static magnetic field exhibit a magnetization along the direction of the main field (B0). Once a radiofrequency pulse is applied, this magnetization vector is tilted towards the transverse plane, and starts to exhibit a special type of rotation around the main field called, precession. Gradient fields are used to spatially encode the magnetization by slightly changing the precession rates as a function of position. One might then conclude that the presence of a foreign object, disturbing the initial magnetization, would consequently result in unexpected precession rates around that object, causing distortion in the decoded image. A simple analogy is to imagine the process of tuning a radio station from transmitted radio-waves. If there is a distortion in either the receiving or the transmission processes, one might either lose the intended station, hear multiple stations at the same time, or hear a wrong station. In terms of MRI artefacts, this is analogous to signal loss, signal pile-up, or geometric distortions, all of which will be collectively defined here as displacements artefacts [23]. For instance, when a 2D slice is selected, the radiofrequency pulse is applied with certain bandwidth around a particular resonant frequency that corresponds to a particular slice position. When distortions in the magnetic field exist, the excited slice will no longer represent the desired image position and signal from various slice positions could either accumulate or not get excited, causing signal pile-up or signal loss. Likewise, during the signal readout each location is expected to be mapped to a resonant frequency and the presence of unexpected magnetic field variations can cause geometric distortions, signal pile-up, or signal loss effects [30].

In addition to displacements artefacts, signal loss also occurs from the rapid dephasing of spins $\left(\mathrm{T}_{2}{ }^{*}\right.$ decay) around the metal objects; i.e., loss of signal coherence within each voxel causing rapid signal decay. Another problem is the failure of some fat suppression techniques that rely on the homogeneity of the magnetic field [31].

\section{Clinical significance: dosimetric impact}

Mapping these artefacts to a brachytherapy application, such as using a titanium applicator, one might expect: 1) the location of the tandem on the MR image might not represent its exact physical location inside the patient in relation to the adjacent organs; 2) signal pile-up and signal loss will occur remarkably around the applicator. The extent of these artefacts will depend on the acquisition parameters, the magnetic field strength, as well as the shape and the orientation of the applicator with respect to the main field [25].

Failing to accurately localize the applicator leads to erroneous identification of the source position during treatment delivery. Accuracy of delivered dose depends on the geometrical accuracy of the source position relative to the target and organs at risk (OARs) [19,24,27]. It has been long established that target coverage and OAR sparing is strongly correlated with clinical outcomes. Hence, applicator displacements or mislocalization can lead to dosimetric errors, which in turn compromise the care for patients $[19,24,26,32,33,34]$. Errors in localizing the applicator can be also reflected as uncertainties 
in the reconstruction process [32]. This results in uncertainties in the dose volume histogram (DVH) parameters for tumor and OARs. Reconstruction uncertainties will be added to other sources of uncertainties in MR-guided workflow such as contouring uncertainties, inter- and intra-fraction variabilities, etc. Tanderup et al. have studied possible systematic uncertainties in applicator reconstruction by simulating shifts in the applicator position (in the order of $2 \mathrm{~mm}$ ) [24,26]. They concluded that DVH parameters will deviate less than $10 \%$ for $90 \%$ of the patients if systematic uncertainties were avoided. Schindel et al. [32] have recently performed a study to simulate the dosimetric impact of applicator displacements as well as the uncertainties of reconstruction. They concluded that in order to avoid more than $10 \%$ change in the prescribed doses, applicator displacements and its reconstruction uncertainties should not exceed $\pm 1.5 \mathrm{~mm}$ and $3 \mathrm{~mm}$, respectively. The displacements were only simulated in the cranial-caudal direction. In another study, $\pm 2 \mathrm{~mm}$ displacements in the anterior-posterior direction were found to have significant dosimetric changes [34]. The dosimetric consequences of applicator shifts in PDR ${ }^{192}$ Ir was studied by DeLeeuw et al. [33]. They found that applicator shifts have larger dosimetric impact on OARs compared to the target. This also matches Tanderup et al. findings, which concluded that rectum and bladder are the most sensitive organs to reconstruction offsets [26], with $\mathrm{D}_{2 \mathrm{~cm}^{3}}$ variations were $5 \pm 1 \% / \mathrm{mm}$ offset in the anterior-posterior direction, where $90 \%$ of the patients have changes $<6 \% / \mathrm{mm}[24,26]$. Nevertheless, it is worth noting that dwell position uncertainty from reconstruction and source positioning for one intracavitary brachyther- apy fraction was estimated in some studies to be $4 \%$ for either OARs $\left(D_{2 \mathrm{~cm}^{3}}\right)$ or the target $\left(D_{90}\right)$ [27].

\section{Metal applicators and needles}

It is well known that MRI is the preferred tool for target definition in GYN applications, especially following GEC-ESTRO Gynecological workgroup recommendations $[18,19,35,36]$. However, when metal applicators/ needles are used in MRI-only workflow, it is important also to identify the extent and the significance of the artefacts as it pertains to the accuracy of applicator reconstruction and the influence on target delineation processes. This need became more prominent when titanium applicators were introduced for MR-guided intracavitary brachytherapy treatment. Titanium applicators are used because they offer greater strength with smaller diameter $(\sim 3.2 \mathrm{~mm})$ than plastic applicators [1]. On the other hand, titanium has higher susceptibility than human tissue $(|\Delta \chi|=\sim 190$ ppm [25]), and the resultant artefacts can be a concern in clinical practice. In particular, displacements artefacts, as described above, could present an obstacle for accurate applicator reconstruction if the apparent location of the applicator tip on the image was shifted from its actual location or if the entire applicator position was misregistered with respect to the adjacent anatomy. This is particularly a concern at high field strength $\geq 3.0 \mathrm{~T}$. In addition, signal pile-up and signal loss could lead to erroneous localization of needles/applicators tip.

Previous effort was made to characterize the artefacts of titanium applicators on $1.5 \mathrm{~T}$ and $3.0 \mathrm{~T}$, as well as to optimize its visualization for accurate reconstruction $[37,38,39,40]$. Phantoms and in vivo results have demon-
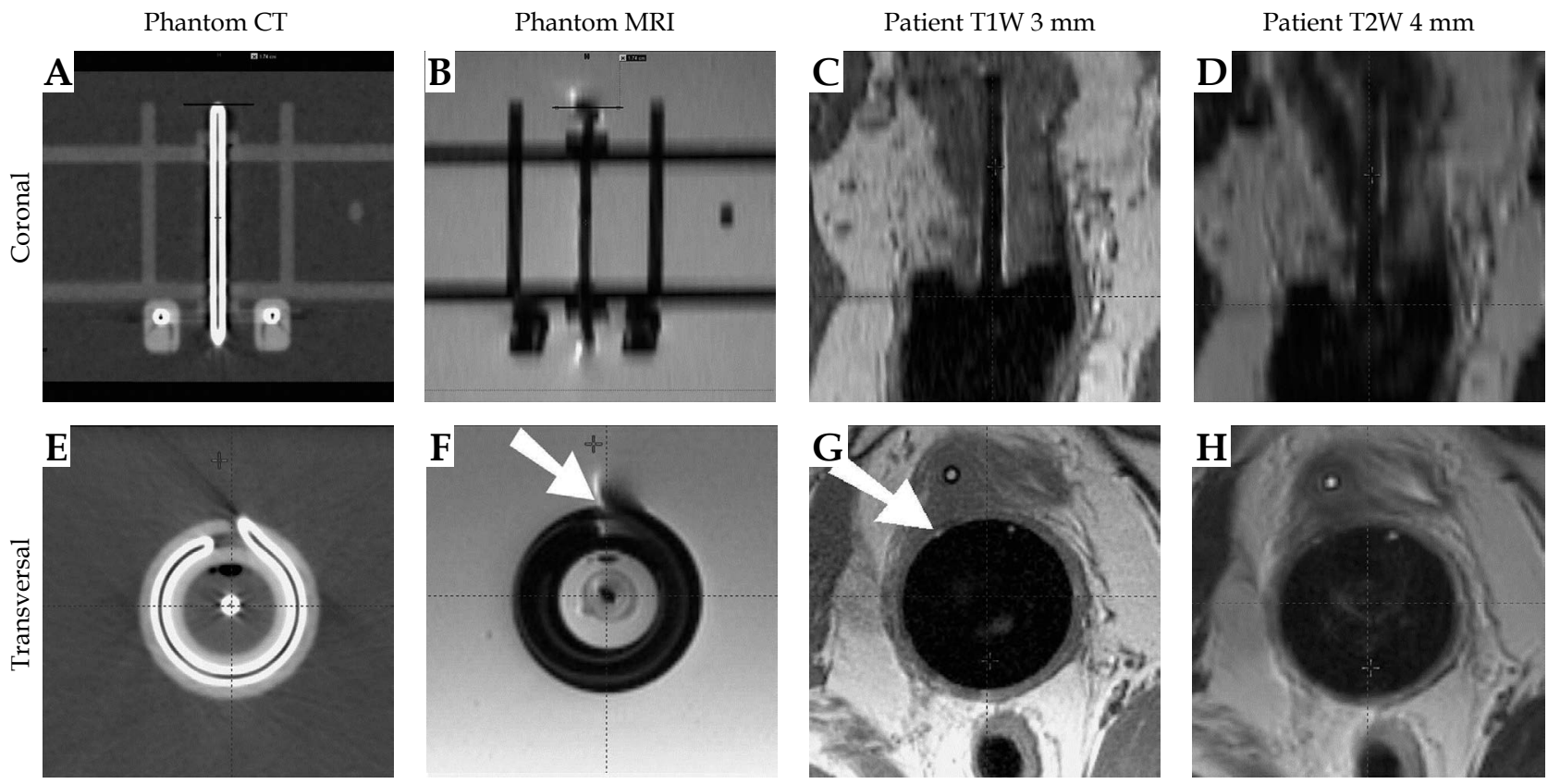

Fig. 1. Adopted from Haack et al. [37], with permission. Coronal (top row) and transversal (bottom row) images showing the titanium applicator in the phantom on computed tomography (CT) images (A and $\mathbf{E}$ ), in phantom on T1-weighted magnetic resonance (MR) images (B and F), patient T1-weighted MR images (C and G), and patient T2-weighted MR images (D and $\mathbf{H})$ - scanned at 1.5 T. The line on (B) shows the position of the applicator tip according to tip position found in the CT image (A). The arrows in (F and $\mathbf{G})$ indicate the artifact used for determining the rotation of the ring 
strated that it is feasible to reconstruct the titanium applicator at $1.5 \mathrm{~T}$ with a mean inter-observer variability of $<1 \mathrm{~mm}[1,37,40]$ (Figure 1). Artefacts could be significantly larger on $3.0 \mathrm{~T}$ (up to $7 \mathrm{~mm}$ for $2 \mathrm{D} \mathrm{T} 2 \mathrm{w}$ fast spinecho [39]), and careful implementation of the MRI-only planning was strongly recommended $[1,27,39]$. If diffusion-weighted imaging (DWI) is to be acquired while titanium applicator is in situ, careful interpretations of the images should be then performed, as signal-pile up/ loss can affect the resultant apparent diffusion coefficient (ADC) maps [2]. The feasibility of DWI on 3.0 T with the titanium applicator in place is questionable [1].

Recently, tungsten-based tandem applicators have been introduced for high-dose-rate (HDR) intracavitary cervical cancer brachytherapy to achieve more conformal dose distribution to non-symmetrical target volumes [41] - so called direction modulated brachytherapy (DMBT) $[41,42,43]$. This new tandem applicator is designed to have 6 peripheral holes instead of a single central channel used in conventional tandems. Tungsten alloy ( $>90 \%$ tungsten) was chosen for its high physical density $\left(\rho=18.0 \mathrm{~g} / \mathrm{cm}^{3}\right)$, allowing better OAR dose sparing than titanium or plastic. Tungsten is also paramagnetic and thus expected to produce metal-related artefacts. Luckily, its susceptibility value is relatively closer to human tissue than that of titanium (i.e., $|\Delta \chi|=\sim 90$ ppm $[25,44]$ ). Preliminary re- sults on phantoms have shown that it produces minimal in-plane artefacts on $1.5 \mathrm{~T}(\sim 0.5 \mathrm{~mm})$ and $3.0 \mathrm{~T}$ (Figure 2) $[45,46,47,48]$. A potential advantage of this new applicator is that it has a tip cap manufactured from PEEK, which has similar susceptibility to water $(|\chi \Delta|=\sim 0.3 \mathrm{ppm})$ [49]. However, this state-of-the-art technology is still developing and commercial TPS reconstruction libraries are not yet available. Further clinical validation is essential to confirm the preliminary findings, particularly in patient studies.

Interstitial titanium needles are also another place where metal artefacts can be seen. In contrast to conventional plastic needles, titanium needles do not bend inside the patient and often result in better implants [19,37,39]. Due to the absence of metal artefacts, plastic needles are a better choice for MRI, however their trajectory cannot be easily predicted within the patients when they bend. On the other hand, the induced artefacts around titanium needles explain the reluctance of adopting them in clinical practice in place of plastic ones, particularly for MRI-only planning. Computed tomography is a better choice to reconstruct titanium needle trajectory, as it can provide a better defined path compared to conventional T2w-MRI $[19,37]$. Displacements artefacts as well as signal fluctuations (e.g., pile-up) around the titanium needle can render its localization challenging, which adds a potential source
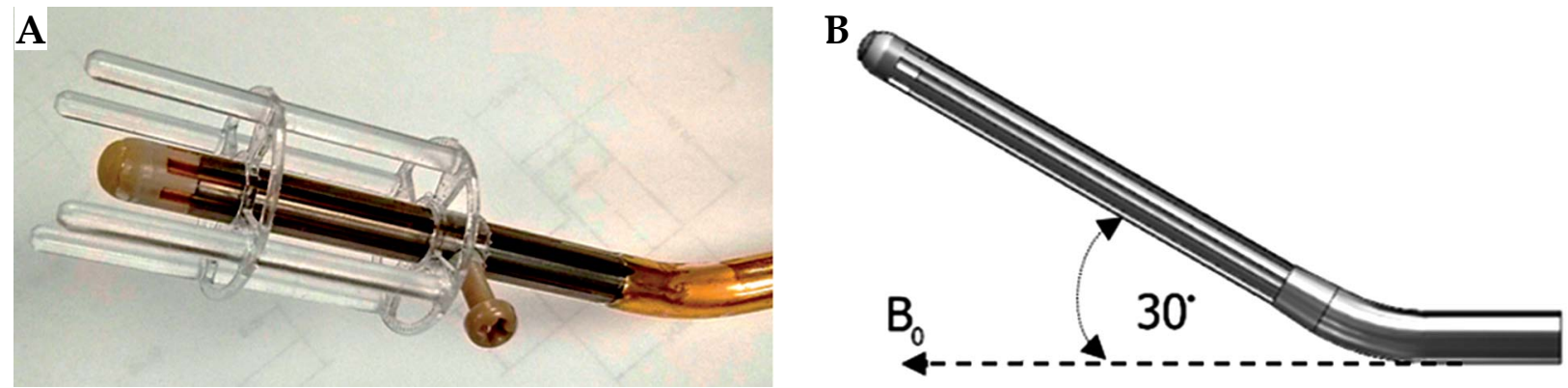

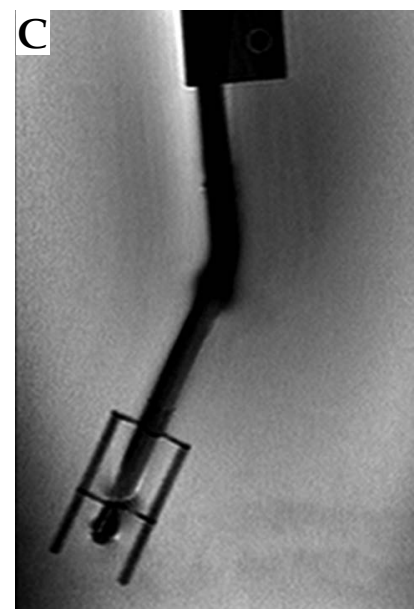

T2w FSE

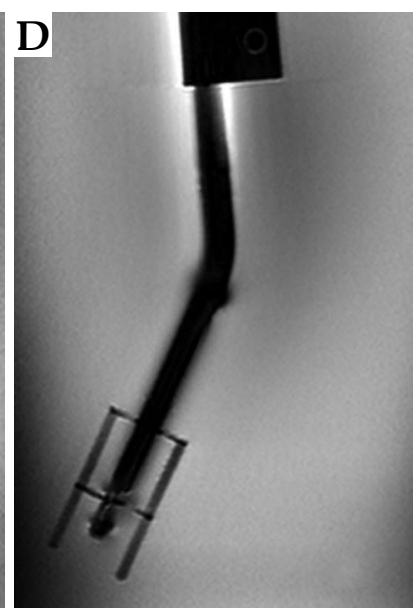

PDw FSE

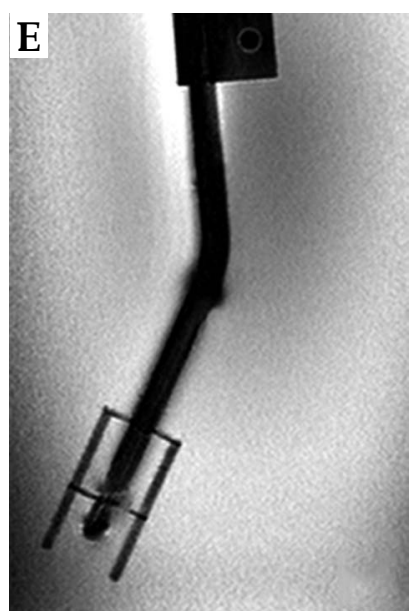

T1w FSE

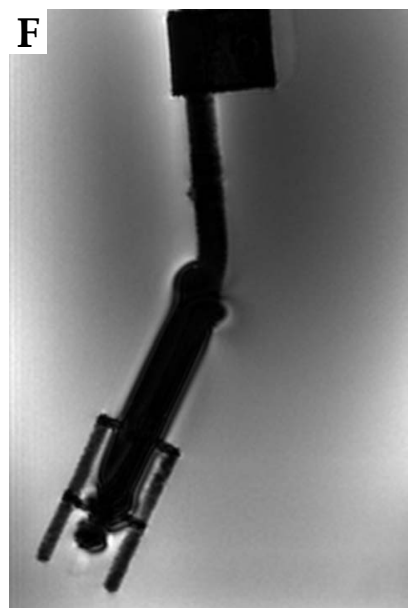

T1w GE

Fig. 2. Figure adopted from Soliman et al. [47], with permission. A) Shows a plastic radial-fiducial with 4 rods - used to visually evaluate the spatial shift - mounted on a prototype of the tungsten-based DMBT tandem applicator. B) shows a sketch of the orientation of the applicator during the scan. The scan was performed in water doped with $\mathrm{MnCl} 2 \mathrm{using}$ four sequences at 3.0 $\mathrm{T}$. C) T2w-FSE: T2-weighted fast spin-echo, D) PDw-FSE: proton density weighted fast spin-echo, E) T1w-FSE: T1-weighted fast spin-echo, and F) T1w-GE: T1 weighted gradient echo 
of uncertainty in calculating DVH parameters $[26,50]$. It might be worth noting that the impact of reconstruction uncertainty for combined interstitial-intracavitary applicator (plastic) was found to be similar to those from tandem-ring applicator except for the sigmoid where interstitial case has slightly larger impact [26].

Nevertheless, signal pile-up from metal needles and applicators could be beneficial in identifying their location in some cases. For instance, Haack et al. utilized the artefacts to guide the reconstruction process of the titanium applicator [37], while Petit et al. employed 3D spoiled gradient-echo (SPGR) in order to distinguish the applicator from the surrounding tissue [40]. Kapur et al. also benefited from the artefacts surrounding the needles to identify them using a 3D fat-suppressed balanced SSFP sequence [51].

An interesting different approach by de Leeuw et al. is to use a dedicated pulse sequence to locate the center of the magnetic field disturbance of the HDR sources [52], and hence allowing a real-time MR-tracking of the sources. The technique was initially implemented to locate prostate permanent seeds implants $[53,54]$, but was recently modified to locate HDR sources. Obviously this approach will have its own technical and logistical challenges. For instance, it requires an MR-compatible afterloader, which is not yet commercially available [52], in addition to the specific technical requirements (pulse sequence, post-processing, etc.) that are not yet available at all centers/vendors.

\section{Imaging sequences for applicator reconstruction}

While GEC-ESTRO guidelines for GYN recommend conventional 2D-T2w fast spin-echo (FSE) for target and OAR delineation with specific parameters $[18,19]$, the recommendations did not highlight optimal MR parameters in terms of applicator reconstruction. Isotropic 3D sequences, however, were recommended as they would allow free reformatting of the slices in any plane during image interpretation. One option for 3D imaging is the 3D T2w FSE with variable flip angle, which has been successfully applied in diagnostic applications $[55,56,57,58]$. A potential aim could be to replace the conventional 2D FSE with one 3D sequence for both target/OAR delineation and applicator reconstruction. For instance, 3D fast-recovery FSE was previously used for this purpose [59]. However, a serious challenge for such approach is the difference in relative tissue contrast in $3 \mathrm{D}$ sequences [55], compared to the conventional contrast provided by 2D sequences, regularly used by clinicians.

It is important to notice here that the methodology of assessing the extent of artefacts of titanium applicator and the accuracy of its reconstruction varied remarkably between groups, particularly from MR imaging perspective. For instance, while some studies highlighted the proton-density FSE sequence to provide better visibility of the applicator [2,38], T1w gradient-echo was selected as a favorable sequence to visualize the applicator in other studies [39]. Petit et al. [40], on the other hand, have used proton-density spoiled gradient-recalled-echo in evaluating the visibility of the titanium Rotterdam applicator.
Sequences also varied between 2D and 3D [1,37,39,40,59]. Additionally, the slice thickness used in these studies varied between 1 and $5 \mathrm{~mm}[27,37,38,39,40,60]$; this affects the level of the out-of-plane artefacts, and consequently the uncertainties of the reconstruction in the slice direction, as well as the SNR of the images on which the reconstruction is performed. Studies also differ in whether or not MRI-markers are used to guide the reconstruction process $[1,2,19,37,38,39,40,61]$.

Advanced pulse sequences to correct for metal artefacts have been developed in the MRI community particularly for diagnostic purposes near metallic implants $[62,63,64,65,66]$. Unfortunately, there has been little interest to optimize these sequences for brachytherapy applications. One main reason might be their lengthy acquisition times. Warner et al. [67] have explored various metal-artefact reduction techniques for brachytherapy planning and concluded that SEMAC [64] was more efficient than the other methods.

While there is not yet an optimal standard sequence to visualize the applicator, there is a clear practical consensus that $\mathrm{T} 2 \mathrm{w}$ sequence is suboptimal for applicator reconstruction compared to other clinical sequences that have shorter echo-time (TE) such as proton-density FSE or T1w FSE.

\section{Summary, recommendations, and future perspectives}

The last decade has seen an increasing interest in understanding technical and clinical challenges accompanying the usage of metal applicators and needles in gynecological brachytherapy. Titanium needles and applicators are preferred over plastic as they offer greater strength at smaller diameters. Tungsten offers an additional advantage, as it allows for non-symmetrical dose distribution, with specific tandem designs, owing to its higher physical density. However, due to the difference in magnetic susceptibilities, the induced metal-artefacts render the accurate localization of metal objects more challenging in MRI-only planning. Phantom and clinical studies demonstrated that conventional 2D T2w FSE is suboptimal in localizing the applicators/needles, particularly when thick slices ( $\geq 4 \mathrm{~mm}$ ) are acquired [37]. Sequences with shorter echo-time (TE) such as PDw have provided better visibility necessary for applicators reconstruction [38].

FSE-based sequences have the advantage over gradient-echo (GRE) sequences of overcoming much of the signal loss occurring from the signal dephasing (incoherence) that is seen around the metal object. However, 3D FSE acquisitions are substantially lengthy, compared to 3D GRE-based sequences that can cover the whole imaging volume in $\leq 5$ minutes [40]. Nevertheless, 3D isotropic resolution can be achieved using an optimized "3D FSE with variable flip angle sequence" in a reasonable scan time $(\leq 10 \mathrm{~min})[18,56,58,59]$. Higher readout bandwidth and higher spatial resolution can reduce geometric and in-plane distortions induced by metal objects. The use of thin slices also reduces the distortions in the slice direction. However, this will also result in lower SNR, partic- 
ularly at low field strength (e.g., 1.5 T). Multiple averages can be then acquired to compensate for SNR loss.

Given the existing variety of pulse sequences for metal-artefacts reduction, the absence of a dedicated sequence for brachytherapy applications is a witness of the still-existing gap between MRI and radiation oncology communities [5]. Current metal-artefacts reduction techniques need yet to be explored and adopted for brachytherapy needs, such as applicator reconstruction. Larger cohort studies to explore the dosimetric impact of the metal artefacts with clinical sequences may be also needed to resolve the clinical concerns regarding the regular usage of metallic applicators/needles.

\section{Disclosure}

Authors report no conflict of interest.

\section{References}

1. Tanderup K, Viswanathan AN, Kirisits C et al. Magnetic resonance image guided brachytherapy. Semin Radiat Oncol 2014; 24: 181-191.

2. Zoberi JE, Garcia-Ramirez J, Hu Y et al. Clinical implementation of multisequence MRI-based adaptive intracavitary brachytherapy for cervix cancer. J Applied Clin Med Phys 2016; 17: 121-133.

3. Banerjee R, Kamrava M. Brachytherapy in the treatment of cervical cancer: a review. Intl J Womens Health 2014; 6: 555-564.

4. Harkenrider MM, Alite F, Silva SR et al. Image-Based Brachytherapy for the Treatment of Cervical Cancer. Int J Radiat Oncol Biol Phys 2015; 92: 921-934.

5. McGee KP, Hu Y, Tryggestad E et al. MRI in radiation oncology: Underserved needs. Magn Reson Med 2016; 75: 11-14.

6. Rivard MJ, Venselaar JLM, Beaulieu L. The evolution of brachytherapy treatment planning. Med Phys 2009; 36: 21362153.

7. Viswanathan AN, Erickson BA. Three-dimensional imaging in gynecologic brachytherapy: a survey of the American Brachytherapy Society. Int J Radiat Oncol Biol Phys 2010; 76: 104-109.

8. Viswanathan AN, Dimopoulos J, Kirisits C et al. Computed tomography versus magnetic resonance imaging-based contouring in cervical cancer brachytherapy: results of a prospective trial and preliminary guidelines for standardized contours. Int J Radiat Oncol Biol Phys 2007; 68: 491-498.

9. Pötter R, Georg P, Dimopoulos JC et al. Clinical outcome of protocol based image (MRI) guided adaptive brachytherapy combined with 3D conformal radiotherapy with or without chemotherapy in patients with locally advanced cervical cancer. Radiother Oncol 2011; 100: 116-123.

10. Nomden CN, de Leeuw AA, Roesink JM et al. Clinical outcome and dosimetric parameters of chemo-radiation including MRI guided adaptive brachytherapy with tandem-ovoid applicators for cervical cancer patients: a single institution experience. Radiother Oncol 2013; 107: 69-74.

11. Mahantshetty U, Swamidas J, Khanna N et al. Magnetic resonance image-based dose volume parameters and clinical outcome with high dose rate brachytherapy in cervical cancers-a validation of GYN GEC-ESTRO brachytherapy recommendations. Clin Oncol 2011; 23: 376-377.

12. Lindegaard JC, Fokdal LU, Nielsen SK et al. MRI-guided adaptive radiotherapy in locally advanced cervical cancer from a Nordic perspective. Acta Oncol 2013; 52: 1510-1519.

13. Chargari C, Magné N, Dumas I et al. Physics contributions and clinical outcome with 3D-MRI-based pulsed-dose-rate intracavitary brachytherapy in cervical cancer patients. Int J Radiat Oncol Biol Phys 2009; 74: 133-139.

14. Owrangi AM, Prisciandaro JI, Soliman A et al. Magnetic resonance imaging-guided brachytherapy for cervical cancer: initiating a program. J Contemp Brachytherapy 2015; 7: 417-422.

15. Villalba RS, Sancho RJ, Palacin OA et al. A new template for MRI-based intracavitary/interstitial gynecologic brachytherapy: design and clinical implementation. J Contemp Brachytherapy 2015; 7: 265-272.

16. Sturdza A, Pötter R, Fokdal LU et al. Image guided brachytherapy in locally advanced cervical cancer: Improved pelvic control and survival in RetroEMBRACE, a multicenter cohort study. Radiother Oncol 2016 [In press].

17. Mongula J, Slangen B, Lambregts D et al. Predictive criteria for MRI-based evaluation of response both during and after radiotherapy for cervical cancer. J Contemp Brachytherapy 2016; 8: 181-188.

18. Dimopoulos JCA, Petrow P, Tanderup K et al. Recommendations from Gynaecological (GYN) GEC-ESTRO Working Group (IV): Basic principles and parameters for MR imaging within the frame of image based adaptive cervix cancer brachytherapy. Radiother Oncol 2012; 103: 113-122.

19. Hellebust TP, Kirisits C, Berger D et al. Recommendations from Gynaecological (GYN) GEC-ESTRO Working Group: Considerations and pitfalls in commissioning and applicator reconstruction in 3D image-based treatment planning of cervix cancer brachytherapy. Radiother Oncol 2010; 96: 153-160.

20. Krishnatry R, Patel FD, Singh P et al. CT or MRI for Imagebased Brachytherapy in Cervical Cancer. Jpn J Clin Oncol 2012; 42: 309-313.

21. Pavamani S, D'Souza DP, Portelance L et al. Image-guided brachytherapy for cervical cancer: a Canadian Brachytherapy Group survey. Brachytherapy 2011; 10: 345-351.

22. Petrič P, Hudej R, Rogelj P et al. Uncertainties of target volume delineation in MRI guided adaptive brachytherapy of cervix cancer: a multi-institutional study. Radiother Oncol 2013; 107: 6-12.

23. Hargreaves BA, Worters PW, Pauly KB et al. Metal-induced artifacts in MRI. AJR Am J Roentgenol 2011; 197: 547-555.

24. Kirisits C, Rivard MJ, Baltas D et al. Review of clinical brachytherapy uncertainties: Analysis guidelines of GEC-ESTRO and the AAPM. Radiother Oncol 2014; 110: 199-212.

25 . Schenck JF. The role of magnetic susceptibility in magnetic resonance imaging: MRI magnetic compatibility of the first and second kinds. Med Phys 1996; 23: 815-850.

26. Tanderup K, Hellebust TP, Lang S et al. Consequences of random and systematic reconstruction uncertainties in 3D image based brachytherapy in cervical cancer. Radiother Oncol 2008; 89: 156-163.

27. Tanderup K, Nesvacil N, Pötter R et al. Uncertainties in image guided adaptive cervix cancer brachytherapy: impact on planning and prescription. Radiother Oncol 2013; 107: 1-5.

28. Shellock FG. MRI Bioeffects, Safety, and Patient Management. Biomedical Research Publishing Group, Playa Del Rey 2014.

29. Schenck JF. Safety of Strong, Static Magnetic Fields. J Magn Reson Imaging 2000; 12: 2-19.

30. Koch KM, King KF, Carl $M$ et al. Imaging near metal: The impact of extreme static local field gradients on frequency encoding processes. Magn Reson Medicine 2014; 71: 2024-2034.

31. Bley TA, Wieben O, François CJ et al. Fat and water magnetic resonance imaging. J Magn Reson Imaging 2010; 31: 4-18.

32. Schindel J, Zhang W, Bhatia SK et al. Dosimetric impacts of applicator displacements and applicator reconstruction-uncertainties on 3D image-guided brachytherapy for cervical cancer. J Contemp Brachytherapy 2013; 5: 250-257.

33. De Leeuw AAC, Moerland MA, Nomden C et al. Applicator reconstruction and applicator shifts in 3D MR-based PDR 
brachytherapy of cervical cancer. Radiother Oncol 2009; 93: 341-346.

34. Yong JS, Ung NM, Jamalludin Z et al. Dosimetric impact of applicator displacement during high dose rate (HDR) Cobalt-60 brachytherapy for cervical cancer: A planning study. Radiation Phys Chem 2016; 119: 264-271.

35. Haie-Meder C, Pötter R, Van Limbergen E et al. Recommendations from Gynaecological (GYN) GEC-ESTRO Working Group (I): concepts and terms in 3D image based 3D treatment planning in cervix cancer brachytherapy with emphasis on MRI assessment of GTV and CTV. Radiother Oncol 2005; 74: 235-245.

36. Pötter R, Haie-Meder C, Van Limbergen E et al. Recommendations from gynaecological (GYN) GEC ESTRO working group (II): concepts and terms in 3D image-based treatment planning in cervix cancer brachytherapy-3D dose volume parameters and aspects of 3D image-based anatomy, radiation physics, radiobiology. Radiother Oncol 2006; 78: 67-77.

37. Haack S, Nielsen SK, Lindegaard JC et al. Applicator reconstruction in MRI 3D image-based dose planning of brachytherapy for cervical cancer. Radiother Oncol 2009; 91: 187-193.

38. Hu Y, Esthappan J, Mutic $S$ et al. Improve definition of titanium tandems in MR-guided high dose rate brachytherapy for cervical cancer using proton density weighted MRI. Radiat Oncol 2013; 8: 16.

39. Kim Y, Muruganandham M, Modrick JM et al. Evaluation of Artifacts and Distortions of Titanium Applicators on 3.0-Tesla MRI: Feasibility of Titanium Applicators in MRI-Guided Brachytherapy for Gynecological Cancer. Int J Radiat Oncol Biol Phys 2011; 80: 947-955.

40. Petit S, Wielopolski P, Rijnsdorp R et al. MR guided applicator reconstruction for brachytherapy of cervical cancer using the novel titanium Rotterdam applicator. Radiother Oncol 2013; 107: 88-92.

41. Han DY, Webster MJ, Scanderbeg DJ et al. Direction-Modulated Brachytherapy for High-Dose-Rate Treatment of Cervical Cancer. I: Theoretical Design. Int J Radiat Oncol Biol Phys 2014; 89: 666-673.

42. Webster MJ, Devic S, Vuong T et al. HDR brachytherapy of rectal cancer using a novel grooved-shielding applicator design. Med Phys 2013; 40: 091704.

43. Webster MJ, Devic S, Vuong $\mathrm{T}$ et al. Dynamic modulated brachytherapy (DMBT) for rectal cancer. Med Phys 2013; 40: 011718.

44. Doty FD, Entzminger G, Yang YA. Magnetism in high-resolution NMR probe design. I: General methods. Concepts in Magn Reson 1998; 10: 133-156.

45. Soliman A, Elzibak A, Fatemi A et al. Comparison of MR Image Quality of Various Brachytherapy Applicators for Cervical Cancer. Med Phys 2015; 42: 3380 (abstract).

46. Soliman A, Elzibak A, Fatemi A et al. Quantitative MRI Assessment of a Novel Direction-Modulated Brachytherapy Tandem Applicator for Cervical Cancer. Med Phys 2015; 42: 3488 (abstract).

47. Soliman A, Han D, Elzibak A et al. MRI Evaluation of a Direction-Modulated Brachytherapy (DMBT) Tandem Applicator for Cervical Cancer On 3T. Med Phys 2016; 43: 3646 (abstract).

48. Soliman AS, Elzibak A, Easton $\mathrm{H}$ et al. Quantitative MRI assessment of a novel direction modulated brachytherapy tandem applicator for cervical cancer at 1.5T. Radiother Oncol 2016 [In press]; DOI: http://dx.doi.org/10.1016/j.radonc. 2016.07.006.

49. Wapler MC, Leupold J, Dragonu I et al. Magnetic properties of materials for MR engineering, micro-MR and beyond. J Magn Reson 2014; 242: 233-242.

50. Rey F, Chang C, Mesina C et al. Dosimetric impact of interfraction catheter movement and organ motion on MRI/CT guided HDR interstitial brachytherapy for gynecologic cancer. Radiother Oncol 2013; 107: 112-116.
51. Kapur T, Egger J, Damato A et al. 3-T MR-guided brachytherapy for gynecologic malignancies. Magn Reson Imaging 2012; 30: 1279-1290.

52. de Leeuw H, Marinus AM, Marco van V et al. A dual-plane co-RASOR technique for accurate and rapid tracking and position verification of an Ir-192 source for single fraction HDR brachytherapy. Phys Med Biol 2013; 58: 7829-7839.

53. de Leeuw H, Seevinck PR, Bakker CJG. Center-out radial sampling with off-resonant reconstruction for efficient and accurate localization of punctate and elongated paramagnetic structures. Magn Reson Med 2013; 69: 1611-1622.

54. Seevinck PR, de Leeuw H, Bos C et al. Highly localized positive contrast of small paramagnetic objects using 3D center-out radial sampling with off-resonance reception. Magn Reson Med 2011; 65: 146-156.

55. Busse RF, Brau ACS, Vu A et al. Effects of refocusing flip angle modulation and view ordering in 3D fast spin echo. Magn Reson Med 2008; 60: 640-649.

56. Mugler JP 3rd. Optimized three-dimensional fast-spin-echo MRI. J Magn Reson Imaging 2014; 39: 745-767.

57. Srinivasan S, Wu HH, Sung K et al. Fast 3DT2-weighted imaging using variable flip angle transition into driven equilibrium (3DT2-TIDE) balanced SSFP for prostate imaging at 3T. Magn Reson Med 2015; 74: 442-451.

58. Takayama Y, Nishie A, Asayama Y et al. Three-dimensional T2-weighted imaging for liver MRI: clinical values of tissuespecific variable refocusing flip-angle turbo spin echo imaging. J Magn Reson Imaging 2015; 41: 339-346.

59. Petric P, Hudej R, Rogelj P et al. 3D T2-weighted fast recovery fast spin echo sequence MRI for target contouring in cervix cancer brachytherapy. Brachytherapy 2008; 7: 109-110.

60. Berger D, Dimopoulos J, Pötter R et al. Direct reconstruction of the Vienna applicator on MR images. Radiother Oncol 2009; 93: 347-351.

61. Schindel J, Muruganandham M, Pigge FC et al. Magnetic resonance imaging (MRI) markers for MRI-guided high-doserate brachytherapy: novel marker-flange for cervical cancer and marker catheters for prostate cancer. Int J Radiat Oncol Biol Phys 2013; 86: 387-393.

62. Cho Z, Kim D, Kim Y. Total inhomogeneity correction including chemical shifts and susceptibility by view angle tilting. Med Phys 1988; 15: 7-11.

63. Koch $\mathrm{K}$, Brau $\mathrm{A}$, Chen $\mathrm{W}$ et al. Imaging near metal with a MAVRIC?SEMAC hybrid. Magn Reson Med 2011; 65: 71-82.

64. Lu W, Pauly KB, Gold GE et al. SEMAC: slice encoding for metal artifact correction in MRI. Magn Reson Med 2009; 62: 66-76.

65. Koch KM, Lorbiecki JE, Hinks RS et al. A multispectral threedimensional acquisition technique for imaging near metal implants. Magn Reson Med 2009; 61: 381-390.

66. den Harder JC, van Yperen GH, Blume UA et al. Off-resonance suppression for multispectral MR imaging near metallic implants. Magn Reson Med 2015; 73: 233-243.

67. Warner L, Kotys-Traughber M, Blume U. MR Imaging Techniques with Reduced Metal Artifact for Improved Brachytherapy Device Visualization. Med Phys 2011; 38: 3427 (abstract).

68. Farvid MS, Ng TWK, Chan DC et al. Association of adiponectin and resistin with adipose tissue compartments, insulin resistance and dyslipidaemia. Diabetes Obes Metab 2005; 7: 406-413. 